\title{
2-D reconstruction of atmospheric concentration peaks from horizontal long path DOAS tomographic measurements: parametrisation and geometry within a discrete approach
}

\author{
A. Hartl, B. C. Song, and I. Pundt \\ Institute of Environmental Physics, University of Heidelberg, Germany \\ Received: 1 August 2005 - Published in Atmos. Chem. Phys. Discuss.: 17 November 2005 \\ Revised: 27 January 2006 - Accepted: 28 January 2006 - Published: 17 March 2006
}

\begin{abstract}
In this study, we theoretically investigate the reconstruction of 2-D cross sections through Gaussian concentration distributions, e.g. emission plumes, from long path DOAS measurements along a limited number of light paths. This is done systematically with respect to the extension of the up to four peaks and for six different measurement setups with 2-4 telescopes and 36 light paths each. We distinguish between cases with and without additional background concentrations. Our approach parametrises the unknown distribution by local piecewise constant or linear functions on a regular grid and solves the resulting discrete, linear system by a least squares minimum norm principle. We show that the linear parametrisation not only allows better representation of the distributions in terms of discretisation errors, but also better inversion of the system. We calculate area integrals of the concentration field (i.e. total emissions rates for non-vanishing perpendicular wind speed components) and show that reconstruction errors and reconstructed area integrals within the peaks for narrow distributions crucially depend on the resolution of the reconstruction grid. A recently suggested grid translation method for the piecewise constant basis functions, combining reconstructions from several shifted grids, is modified for the linear basis functions and proven to reduce overall reconstruction errors, but not the uncertainty of concentration integrals. We suggest a procedure to subtract additional background concentration fields before inversion. We find large differences in reconstruction quality between the geometries and conclude that, in general, for a constant number of light paths increasing the number of telescopes leads to better reconstruction results. It appears that geometries that give better results for negligible measurement errors and parts of the geometry that are better resolved are also less sensitive to increasing measurement errors.
\end{abstract}

Correspondence to: A. Hartl

(andreas.hartl@iup.uni-heidelberg.de)

\section{Introduction}

The importance of knowing exact amount and distribution of trace gases in the atmosphere on a global scale has become more and more evident over the past decades. But knowledge of regional or local concentration distributions is indispensable when it comes to assessing the quality of chemical transport models on microscales or quantifying total emissions of sources and distinguishing between them. Measurements of trace gases are either point measurements providing concentration values on site, and thus being sensitive to local and temporal fluctuations, or remote sensing, yielding path integrated mean values. Combining path integrating measurements along several paths and tomographic retrieval methods, 2- or 3-dimensional concentration fields can be obtained.

So far, a variety of studies was dedicated to the remote sensing of indoor gas concentrations and their dispersion by different experimental techniques, improving the time resolution of the measurement procedure and quality of the reconstructed 2-D concentration maps (e.g. Yost et al., 1994; Drescher et al., 1997; Fischer et al., 2001). A remote sensing technique especially suited for trace gases in the atmosphere is the Differential Optical Absorption Spectroscopy (DOAS) (e.g. Platt, 1994), which allows retrieval of light path integrated concentrations of a large number of species such as $\mathrm{NO}_{2}, \mathrm{O}_{3}, \mathrm{SO}_{2}, \mathrm{HCHO}, \mathrm{HONO}, \mathrm{BrO}$ and aromatic compounds (e.g. benzene and toluene). Active DOAS measurements use telescopes with artificial light sources and retroreflectors to redirect the light beams back to the telescope (so called Long Path (LP)-DOAS), where path lengths vary between several hundred meters up to $20 \mathrm{~km}$. First tomographic measurements employing the LP-DOAS technique were carried out to investigate the emissions of a motorway (Pundt et al., 2005). Using two telescopes and 16 light paths, the $\mathrm{NO}_{2}$ concentration field perpendicular to the motorway was retrieved and within all errors results are in good agreement

Published by Copernicus GmbH on behalf of the European Geosciences Union. 
with model expectations (Laepple et al., 2004). This study used a discrete approach by approximating the $\mathrm{NO}_{2}$ concentration field by a finite sum over local piecewise constant (so called box) and piecewise linear basis functions, respectively. The state vector of discrete concentration values was fitted to the measurement data by a least squares minimum norm principle, comparing three iterative projection algorithms originally developed to solve large systems of equations in image reconstruction: The Algebraic Reconstruction Technique (ART), the Simultaneous ART (SART) and the Simultaneous Iterative Reconstruction Technique (SIRT), which were already studied in Todd and Ramachandran (1994a) for 2D reconstruction of Gaussian shaped indoor gas concentrations. Both studies find that ART performs best, if measurement errors are negligible, whereas SIRT is preferred for noisy data. Instead of choosing the least squares solution minimising the quadratic constraint of the state vector norm, the Twomey-Tikhonov approach (e.g. Twomey, 1996), introduced by Twomey for retrieval of atmospheric profiles, controls the weight of some quadratic constraint by a parameter that has to be chosen. Often not the norm of the state vector itself, but its gradient is chosen to influence the smoothness of the solution. Price et al. (2001) use the norm of the third derivative to make the reconstructed distributions of indoor gas concentrations locally quadratic (Low Third Derivative (LTD)-method) and point out that in their case the positivity constraint on the reconstructed concentration values can be almost neglected so that the method becomes linear and, by one matrix multiplication, very fast. Other discrete algorithms from image reconstruction seek for the most probable reconstruction by maximising some measure of likelihood or expectation that involves logarithmic functionals instead of quadratic constraints. Samanta and Todd (2000) find the methods Maximum Likelihood with Expectation Maximisation (MLEM) and the Multiplicative Algebraic Reconstruction Technique (MART) to give less noisy results than ART but stress the fact that performance may depend significantly on the individual distributions considered and that it is very sensitive to measurement errors. In a further study (Verkruysse and Todd, 2004) MLEM is combined with a new grid translation method (for box discretisation) that takes into account several reconstruction grids shifted against each other in the plane to improve the reconstruction of concentration peaks. In principle, global basis functions extending over the whole area or functions depending on further parameters to be fitted are possible. In fact, Price et al. (2001) claim to get better results for the reconstruction of indoor gas concentrations, compared to ART, by using a method proposed by Drescher (1996) where a certain number of Gaussians with variable variances, peak locations and heights and orientations are fitted to the data using a least squares criterion. But as this method is highly nonlinear, unclear in its convergence and needs considerable calculation time, it does not seem very favourable to be used for extensive simulations, e.g. studying sensitivity to measurement errors or investigating different geometries. Furthermore, it contains unavoidable strong functional a priori and was reported to fail for the reconstruction of the motorway emission plume with steep concentration gradients in Laepple et al. (2004).

For atmospheric measurements in particular, the resolution of the reconstructed distribution is limited by the number of light paths of the measurement configuration, while the duration of the measurement cycle for time dependent distributions has large influence on the reconstruction error and is due mainly to successive scanning of the individual paths by the emitting system. Conventional LP-DOAS instruments emit one light beam only and are not very easily pointed at different targets. Here we refer to an indoor experiment (Mettendorf et al., 2006) designed to evaluate the properties of a novel instrument which emits up to six beams at once (Pundt and Mettendorf, 2005). Three telescopes emitting four beams were used to cover a horizontal area of $15 \times 10 \mathrm{~m}^{2}$ regularly by three $90^{\circ}$-beam fans sitting in the corners of the area with 39 light paths in total $(3 \times 12$ light paths plus one additional per telescope for instrumental reasons that are irrelevant for atmospheric measurements. Effectively, this geometry corresponds to the one of Fig. 1c with 36 light paths). One or two cylinders (radius $=1 \mathrm{~m}$ ), filled with $\mathrm{NO}_{2}$, were placed into this area to simulate locally confined concentration fields, e.g. plumes emitted by a point source.

In this work, we systematically investigate the 2-D reconstruction of atmospheric trace gas distributions on the background of this experiment for future atmospheric measurements. That is, we consider geometries with a relatively small total number of light paths (36), peripherically positioned retro-reflectors and locally confined distributions. We think of horizontal monitoring of the atmosphere, but all results apply to vertical sections as well. Following the semiempirical approach that describes turbulent transport and advection of gas in the atmosphere by a Gaussian diffusion model, we assume Gaussian exponentials as test distributions. We prefer to use a physically intuitive (if possible linear) reconstruction scheme that allows controlled implementation of a priori information or constraints for atmospheric measurements, for example, if path integrating measurements are supported by in situ measurements. In the case of peaks located arbitrarily in the measurement area, the least squares approach minimising the norm of the state vector rather than its derivatives seems plausible, but for extended, smooth concentration fields this could be different, especially if information on atmospheric concentration gradients is available. We use ART and SIRT to obtain the least squares minimum norm solutions. It is known that for isolated concentration peaks reconstruction grids can be chosen that lead to highly under-determined systems. This is investigated in detail by calculating discretisation, inversion and total reconstruction errors separately for varying peak extensions, comparing piecewise constant and piecewise linear basis functions (Sect. 4.1). Additionally to commonly 
used quality criteria of the reconstruction, we estimate reconstructed area integrals of the concentration field, again depending on the peak extensions (Sect. 4.2). On the basis of our results we suggest a reconstruction procedure if a smooth background concentration is present (Sect. 4.3). The above mentioned grid translation scheme is adopted for linear basis functions in two different ways and discussed for varying extensions. Finally, different geometries are compared (Sect. 4.4). They differ from the ones studied in Todd and Ramachandran (1994b) and Todd and Bhattacharyya (1997) in that we consider a number of light paths many times smaller and which is the same for all geometries. Furthermore, our geometries do not include mirrors to generate parallel projections. Additional to simulations, we use arguments from linear inversion theory to evaluate measurement setups for an expected trace gas distribution and different levels of noise.

\section{Reconstruction method}

\subsection{Discretisation}

Results of an LP-DOAS analysis are direct integrals of atmospheric concentrations $c(\mathbf{r})$

$d_{i}=\int_{i} d s c(\mathbf{r}) \quad i=1, \ldots, m$,

where $i$ numbers the light paths and $d_{i}$ are the so called column densities. Direct analytic inference of $c(\mathbf{r})$ common in medical applications and used by Wolfe and Byer (1982) in a first proposal for tomographic measurements in air is not applicable in our case of low and irregular spatial coverage, so strategies usually amount to some kind of fit procedures. One approach, also very common in computerised tomography, is to represent $c(\mathbf{r})$ by a sum over $n$ "basis functions" $b_{j}$ with local support

$c(\mathbf{r}) \approx \sum_{j=1}^{n} x_{j} b_{j}(\mathbf{r})$,

thus leading to the linear system of equations

$\mathbf{A x}=\boldsymbol{d}$,

$\boldsymbol{x}$ and $\boldsymbol{d}$ being the state and data vector with components $x_{i}$ and $d_{i}$, respectively.

Parametrisation by piecewise constant basis functions (box functions or box basis) - being nonzero only in one pixel and vanishing anywhere else - is used when resolution is high and calculation time plays a major role. $A_{i j}$ then stores the lengths of path $i$ in pixel $j$. With large pixels and small systems of equations, piecewise linear basis functions interpolating linearly between nodes of the discretisation grid, as discussed in Ingesson et al. (1998) and used in Laepple et al. (2004) reduce discretisation errors. $x_{j}$ now is the concentration value of the reconstructed distribution on grid node $j$.
2.2 Least squares minimum norm solution, weighting and Bayesian a priori

In practice, Eq. (3) cannot be solved at all, not uniquely or the unique solution might be unstable. It is straightforward to use a constrained least squares principle for the data residuum $\left((\boldsymbol{d}-\mathbf{A} \boldsymbol{x})^{T}(\boldsymbol{d}-\mathbf{A} \boldsymbol{x})\right)^{1 / 2}$ instead:

$(\boldsymbol{d}-\mathbf{A} \boldsymbol{x})^{T} \mathbf{W}(\boldsymbol{d}-\mathbf{A} \boldsymbol{x})=\min ! \quad \boldsymbol{x}>\mathbf{0}$,

where weighting is included by the matrix $\mathbf{W}$. In the underdetermined case, usually additional constraints will be necessary to pick a unique solution. As mentioned, for peak distributions we choose the solution with smallest norm (as no other information about the concentration field is available), i.e. we demand

$\boldsymbol{x}^{T} \boldsymbol{x}=\min !$,

which just produces least squares minimal norm solutions. With an a priori guess $\boldsymbol{x}_{a}$ different from zero Eq. (5) is replaced by

$\left(\boldsymbol{x}-\boldsymbol{x}_{a}\right)^{T}\left(\boldsymbol{x}-\boldsymbol{x}_{a}\right)=\min !$

Iterative projection algorithms converging to least squares solutions of the above kind have been studied extensively in image reconstruction. ART applies corrections of the data residuum sequentially corresponding to the rows in Eq. (3), starting with a given $\boldsymbol{x}^{(0)}$, and is reported to work best when no noise is present. SART and SIRT, applying corrections simultaneously for all rows, are better when noise is present but converge slower than sequential methods. The a priori $\boldsymbol{x}_{a}$ is provided by the iteration start $\boldsymbol{x}^{(0)}$. The order of the algorithms according to (Todd and Ramachandran, 1994a) and (Laepple et al., 2004) is ART $>$ SART $>$ SIRT, reversing to SIRT $>$ SART $>$ ART with noise in both studies. Here, we do not go into the details of the iteration procedures themselves but refer the reader to the two studies mentioned and references therein and in the following will only consider ART and SIRT. We just would like to mention that stopping the iteration process prematurely can be used to regularise the solution, especially in the presence of measurement noise.

We would like to emphasise that these algorithms do not actually solve Eqs. (4) and (5) or (6) but rescaled versions, something not always made clear in the literature. In Trampert and Leveque (1990) it is pointed out that SIRT and SART without positivity constraint minimise $(\boldsymbol{d}-\mathbf{A} \boldsymbol{x})^{T} \mathbf{A}_{2}{ }^{-1}(\boldsymbol{d}-\mathbf{A} \boldsymbol{x})$ where for the under-determined case the solution closest to $\boldsymbol{x}^{(0)}$ with respect to the norm $\boldsymbol{x}^{T} \mathbf{A}_{1} \boldsymbol{x}$ is generated. Here

$$
\begin{aligned}
& \mathbf{A}_{\mathbf{1}}=\operatorname{diag}\left(\sum_{i=1}^{m} A_{i j}^{\alpha}\right) \quad \mathbf{A}_{\mathbf{2}}=\operatorname{diag}\left(\sum_{j=1}^{n} A_{i j}^{2-\alpha}\right) \\
& 0 \leq \alpha \leq 2
\end{aligned}
$$

represent a one parameter family of algorithms with $\alpha=0$ for SIRT, $\alpha=1$ for SART. Referring to the box basis, this means 
that SART weights light paths with the inverse of their length and pixels with the partial light path lengths added up in the pixel. SIRT does not weight pixels whereas light paths get weights inverse to the square of the partial path lengths. The convergence of ART is more involved (e.g. Natterer, 1986) but corresponds to SIRT in the consistent case. Favouring better sampled pixels makes sense, but weighting paths with the inverse of their lengths is somehow contradictive to the fact that, in general, relative DOAS measurement errors decrease with increasing path length. Whether weighting does play a role, though, depends on the problem being consistent and/or over- or under-determined. In the under-determined case one gets

$\boldsymbol{x}=\boldsymbol{x}^{(0)}+\mathbf{A}_{\mathbf{1}}^{-1} \mathbf{A}^{T}\left(\mathbf{A A}_{\mathbf{1}}{ }^{-1} A^{T}\right)^{-1}\left(\boldsymbol{d}-\mathbf{A} \boldsymbol{x}^{(0)}\right)$

(provided the inverse exists) without any weighting of the light paths. In the generalised least squares formalism, again $\boldsymbol{x}^{(0)}$ represents the a priori $\boldsymbol{x}_{a}, \mathbf{A}_{\mathbf{1}}$ its covariance and the error covariance is zero. In the pure least squares solution no a priori in the above sense enters.

\subsection{Grid translation}

The key idea of this method suggested in Verkruysse and Todd (2004) is that for irregular light path geometries the choice of the discretisation grid is to some extent arbitrary and (especially for narrow peaks) in general not optimal. Therefore, the authors suggest using several reconstruction grids that are generated by shifting the initial grid in both directions and combine the results on a grid of higher spatial resolution. Here, this scheme is adopted for linear basis functions and regular grids in the following way: If there are $m_{x}\left(m_{y}\right)$ shifts in $x(y)$ direction, the first grid is generated by shifting the original nodes by $l_{x} /\left(m_{x}+1\right)$ in $x$ direction $\left(l_{x}\right.$ : pixel width in $\left.x\right)$. This is performed $m_{x}$ times, then the initial grid is shifted by $l_{y} /\left(m_{y}+1\right)$ in $y$ direction and the procedure is repeated, and so forth. This results in $M=\left(m_{x} m_{y}+m_{x}+m_{y}+1\right)$ reconstructions and $N=\left[\left(m_{x}-1\right)\left(n_{x}+1\right)+1\right] \times\left[\left(m_{y}-1\right)\left(n_{y}+1\right)+1\right]$ nodes of a high resolution grid, where $n_{x}\left(n_{y}\right)$ is the number of nodes of the initial grid in $x(y)$ direction. Two different schemes to get the state vector $\boldsymbol{X}$ of the high resolution grid from the individual reconstructions are considered: In a composite scheme, the component $X_{j}$ for grid node $\mathbf{r}_{j}$ is taken from the shifted grid $i$ that has a coinciding node there

$X_{j}=x_{j}^{i}$.

As the boundary grid lines do not change, here the average of coinciding nodes is taken. In the averaging scheme $X_{j}$ is taken as the average of all individual reconstructions on its grid nodes $\mathbf{r}_{j}$ :

$X_{j}=\frac{1}{M} \sum_{i=1}^{N} c_{\mathrm{rec}}^{i}\left(\mathbf{r}_{j}\right)$

\subsection{Reconstruction errors}

For atmospheric tomography, the concentration field $c_{\mathrm{rec}}^{\varepsilon}(\mathbf{r})=\sum_{j} x_{j}^{\varepsilon} b_{j}(\mathbf{r})$, reconstructed from error $(\varepsilon)$ afflicted data, will always differ from the real, unknown concentration $c(\mathbf{r})$. The reconstruction error field can be written as

$$
\begin{aligned}
& \Delta c^{\varepsilon}(\mathbf{r})=c(\mathbf{r})-c_{\mathrm{rec}}^{\varepsilon}(\mathbf{r}) \\
& =\underbrace{c(\mathbf{r})-\sum_{j} x_{j}^{i d} b_{j}(\mathbf{r})}_{\Delta \operatorname{disc}(\mathbf{r})}+\underbrace{\sum_{j} x_{j}^{i d} b_{j}(\mathbf{r})-\sum_{j} x_{j} b_{j}(\mathbf{r})}_{\Delta \operatorname{inv}(\mathbf{r})} \\
& +\underbrace{\sum_{j} x_{j} b_{j}(\mathbf{r})-\sum_{j} x_{j}^{\varepsilon} b_{j}(\mathbf{r})}_{\Delta \varepsilon(\mathbf{r})} .
\end{aligned}
$$

Here, the discretisation error $\Delta \operatorname{disc}(\mathbf{r})$ arises because, in general, $c(\mathbf{r})$ cannot be perfectly represented by the basis functions. $\boldsymbol{x}^{i d}$ is the best possible representation, e.g. in a least square sense, for a given grid. Even if the discretisation error happens to be very small, the reconstruction result will differ from the real field. We call this difference $\Delta \operatorname{inv}(\mathbf{r})$ the inversion error. Finally, the measurement error will change the result of an ideal measurement, which leads to the term $\Delta \varepsilon(\mathbf{r})$. If the reconstruction is linear and given by the matrix $\mathbf{A}^{\text {inv }}$, then propagation of the measurement error with covariance $\mathbf{S}_{\varepsilon}$ can be expressed analytically:

$$
\begin{aligned}
& \operatorname{var}_{\varepsilon}\left\{c_{\mathrm{rec}}^{\varepsilon}\right\}(\mathbf{r})=\operatorname{var}_{\varepsilon}\left\{\Delta c_{\mathrm{rec}}^{\varepsilon}\right\}(\mathbf{r}) \\
& =\sum_{i, j}\left(\mathbf{A}^{\mathrm{inv}} \mathbf{S}_{\varepsilon}\left(\mathbf{A}^{\mathrm{inv}}\right)^{T}\right)_{i j} b_{i}(\mathbf{r}) b_{j}(\mathbf{r}) .
\end{aligned}
$$

This means additional contributions for correlated measurement errors and overlapping basis functions, like the linear basis functions.

A straightforward calculation shows that, if

$$
\begin{aligned}
& \boldsymbol{x}^{i d} \text { minimises }\left\|c(\mathbf{r})-\sum_{j} x_{j} b_{j}(\mathbf{r})\right\| \\
& :=\sqrt{\int d A\left(c(\mathbf{r})-\sum_{j} x_{j} b_{j}(\mathbf{r})\right)^{2}},
\end{aligned}
$$

then for the expectation values $E$ the following relation holds

$$
E_{\varepsilon}\left\{\left\|\Delta c^{\varepsilon}(\mathbf{r})\right\|^{2}\right\}=\|\Delta \operatorname{disc}(\mathbf{r})\|^{2}+\|\Delta \operatorname{inv}(\mathbf{r})\|^{2}+E_{\varepsilon}\left\{\|\Delta \varepsilon(\mathbf{r})\|^{2}\right\},
$$

which means that the integrated contributions add up quadratically.

\subsection{Reconstruction quality}

To find the best parameters for the reconstruction and compare different algorithms, usually an ensemble $(e)\left\{c^{e}(\mathbf{r})\right\}$ of realistic test distributions is considered and some quality criterion is optimised for the ensemble mean. Working with 
continuous fields, we use the integral of the squared reconstruction error, normalised to give 1 if the reconstructed field is just the constant average of the test distribution. This number, representing the overall reconstruction error, is known as nearness in image reconstruction:

nearness $=\frac{\left\|\Delta c^{e}\right\|}{\left\|c^{e}-\bar{c}^{e}\right\|}$.

Also the Pearson $r^{2}$-correlation factor between a suitable number of image pixels of the test and reconstructed distribution can be used. We found that, in general, both lead to the same results. To get more descriptive measures for the reconstruction quality, we furthermore introduce the following relative numbers:

$R T I=$ ratio of the total area integrated concentration

$=\frac{\int_{\text {Area }} d A c_{\mathrm{rec}}^{e}}{\int_{\text {Area }} d A c^{e}}$,

$R P I=$ ratio of the concentration integrals

within the peak (plume)

$=\frac{\int_{O} d A c_{\mathrm{rec}}^{e}}{\int_{O} d A c^{e}}$.

In the case of Gaussians, we take $3 \times \max \left(\sigma_{x}, \sigma_{y}\right)$ as the radius of the test distribution (at $3 \sigma$ the peak value has fallen to $1 \%$ ). For the reconstructed distribution, we add one pixel length of the reconstruction grid to this radius to take account of the spreading within the pixels for the box basis and the interpolation between neighbouring grid points for the linear basis functions, respectively. This, especially in the case of several large distributions with overlap and for larger pixel sizes, will only be a rough estimate. If the wind speed has a component perpendicular to the reconstruction area, $R P I$ provides a measure for the precision of emission rates or total emissions. For horizontal cuts through the atmosphere this is the case, e.g., if the area contains a source with advective vertical transport. For horizontal transport of a plume this number can be related to total emissions only with further assumptions on the vertical dispersion since its release.

In the context of air quality regulation one might be interested in maximum values of the concentration, so we also use the

relative peak difference $=$ relative difference of maximum

peak values between original and reconstructed distribution

$=\left(\max c_{\mathrm{rec}}^{e}-\max c^{e}\right) / \max c^{e}$.

\section{Evaluation of the measurement geometry}

In a discrete approach, geometry and discretisation grid are linked in the matrix $\mathbf{A}$. While the discretisation error is given by the resolution of the discretisation, the inversion error strongly depends on the geometry. Even if $\mathbf{A}$ can be inverted, the result still depends on its conditioning and choice of the inversion algorithm. In the under-determined case components of $\boldsymbol{x}$ in the null space of $\mathbf{A}$ are fixed by a priori information or constraints. Evaluation of the geometry is now inherently linked to the reconstruction method and, if nonlinear, not even independent of the concentration distribution. Then criteria like Eq. (15) have to be evaluated for a suitable ensemble.

The singular value decomposition is helpful to investigate the null space of $\mathbf{A}$. Writing $\mathbf{A}=\mathbf{U} \Lambda \mathbf{V}^{T}$ with orthonormal matrices $\mathbf{U}(m \times m)$ and $\mathbf{V}(n \times n)$ and $\Lambda=\operatorname{diag}\left(\lambda_{1}, \ldots, \lambda_{\operatorname{rank}(\mathbf{A})}, 0, \ldots\right), \boldsymbol{x}$ can be uniquely represented by the column vectors of $\mathbf{V}: \boldsymbol{x}=\sum_{j=1}^{n} \tilde{x}_{j} \boldsymbol{v}_{j}$, where $\mathbf{v}_{j}$ with $j>\operatorname{rank}(\mathbf{A})$ span the null space. For a component $j>\operatorname{rank}(\mathbf{A})$ of $\boldsymbol{x}$ the nullspace does not contribute, if $\tilde{x}_{j}=0$. On the other hand, if $\sum_{j>\operatorname{rank}(\mathbf{A})} V_{i j}{ }^{2}=0$, values for $x_{i}$ can be (and for the least squares solution are) completely fixed by the data. In fact, the latter sum is just $(1-\mathbf{R})_{i i}, \mathbf{R}$ being the resolution matrix (averaging kernel) that relates the true and reconstructed state via $\boldsymbol{x}=\mathbf{R} \boldsymbol{x}_{\text {true }}=\mathbf{A}^{\mathrm{inv}} \mathbf{A} \boldsymbol{x}_{\text {true }}$, here for the generalised inverse $\mathbf{A}^{\mathrm{inv}}$ of $\mathbf{A}$ which is equivalent to the unweighted least squares minimum norm solution with uniform a priori covariance $\mathbf{S}_{\mathbf{a}}$.

The probabilistic interpretation of the generalised least squares formalism at least allows to get an idea of how different geometries perform on fairly general assumptions by choosing the a priori model correspondingly and for different levels of noise. In Rodgers (2000) the quantity

$d_{s}=\operatorname{trace}(\mathbf{R})$

with $\mathbf{R}$ the resolution matrix of the generalised least squares solution, is interpreted as the number of degrees of freedom of the signal against the noise, i.e. the number of independent quantities that can be measured. For the unweighted least squares minimum norm solution with uniform $\mathbf{S}_{\mathbf{a}}$ one just has $\operatorname{trace}(\mathbf{R})=\operatorname{rank}(\mathbf{A})$. Alternatively

$H=-1 / 2 \log _{2} \operatorname{det}(1-\mathbf{R})$

is given as a measure of the number of distinct states distinguishable by the measurement.

\section{Results and discussion}

In the following, we consider a square area of $100 \times 100$ arbitrary units (a.u.) that is crossed by 36 light beams. This could be realised, for example, by three telescopes emitting six rays each and scanning two different geometries. Six geometries (Fig. 1) are investigated. They consist of two, three or four telescopes generating either $90^{\circ}$ - or $180^{\circ}$-beam fans. The light paths were chosen to cover the area more or less regularly without claiming to present the ideal solution, for example with respect to the number of retro-reflectors, which varies between 25 and 36 for the geometries shown. 


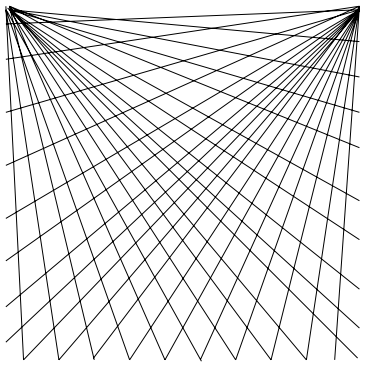

a) $2 \mathrm{~T} 90^{\circ}$

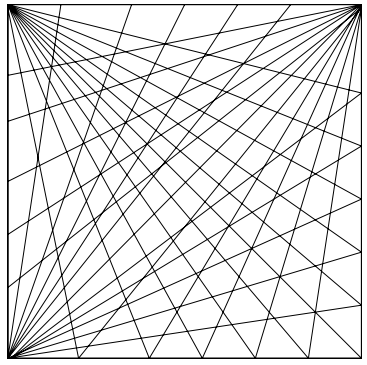

c) $3 \mathrm{~T} 90^{\circ}$

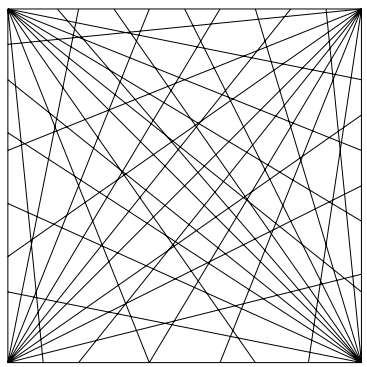

e) $4 \mathrm{~T} 90^{\circ}$

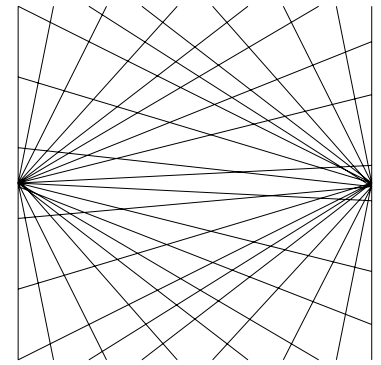

b) $2 \mathrm{~T} 180^{\circ}$

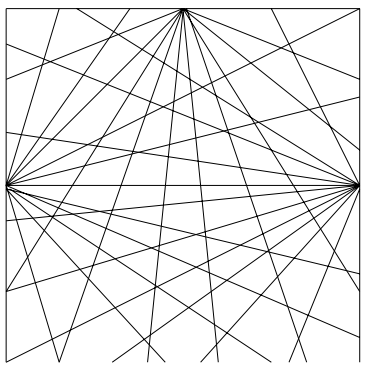

d) $3 \mathrm{~T} 180^{\circ}$

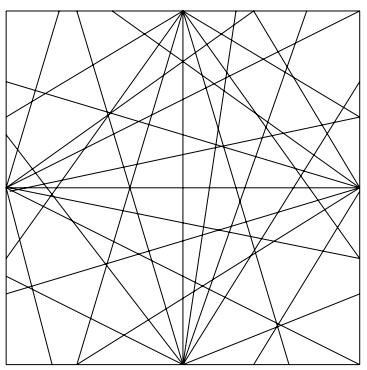

f) $4 \mathrm{~T} 180^{\circ}$

Fig. 1. Geometries with two $(\mathbf{a}, \mathbf{b})$, three $(\mathbf{c}, \mathbf{d})$ and four $(\mathbf{e}, \mathbf{f})$ telescopes with light paths emitted in $90^{\circ}$ (left column)- or $180^{\circ}$ (right column)-fans. The square area is $100 \times 100$ a.u. All geometries consist of 36 light paths. Geometry (c) corresponds to the one used for the indoor experiment reported in Mettendorf et al. (2006).

Table 1. Variances and extensions of the Gaussian test distributions. The radius of the extension is defined by $3 \sigma$, where the exponential has fallen to $1 \%$ of its peak value.

\begin{tabular}{cc}
\hline ensemble & $\sigma$ [a.u.] \\
\hline 1 & $3 \ldots 5$ \\
2 & $5 \ldots 10$ \\
3 & $10 \ldots 20$ \\
4 & $20 \ldots 30$ \\
\hline
\end{tabular}

For the test distributions, we take up to four Gaussians of variable variances and maximum values, located randomly in the area. The Gaussians are divided into four ensembles (Table 1). Ensemble 1 represents the lower limit of narrow peaks

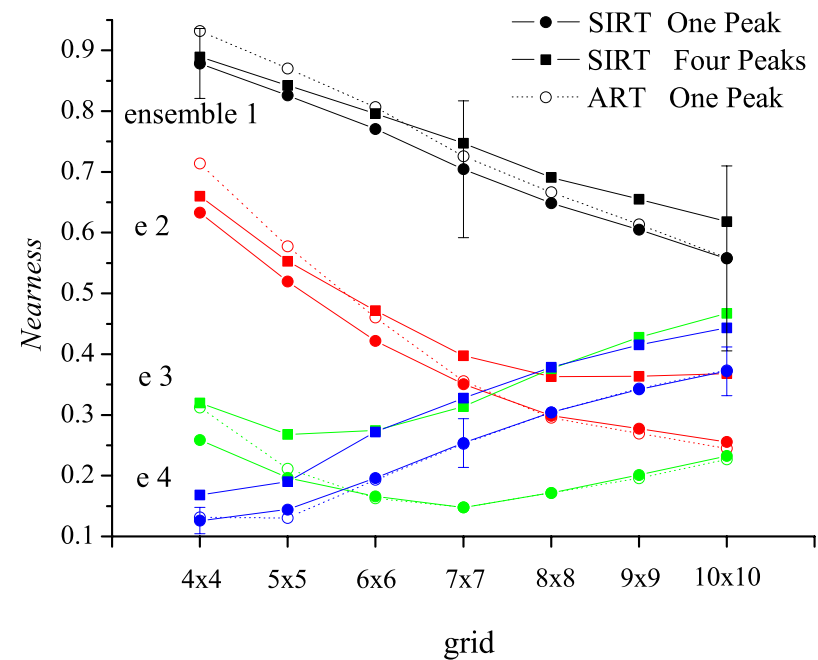

Fig. 2. Ensemble mean nearness for geometry $3 \mathrm{~T} 90^{\circ}$ versus grid dimension, using linear basis functions. $n \times n$ refers to the number of pixels. Variances shown for illustration are for one peak, reconstructed by SIRT. For four distributions peak maxima vary between 0.1 and 1 a.u.

that are still detectable for our coverage with light paths. Here the aim is rather to locate the peaks and to reconstruct total amounts of concentration, whereas for extended peaks of ensemble 3 and 4 reconstruction of the actual distribution is feasible. Reconstructing more than four peaks is not reliable with our number of light paths, especially if artefacts are similar in size to the original peaks.

In the following, we do not make use of any explicit weighting, as, except for a short discussion in Sect. 4.4, measurement errors are not taken into account. First, basic observations will be presented for the specific geometry labeled $3 \mathrm{~T} 90^{\circ}$ that was realised in the indoor experiment. The last section contains a comparison with other geometries.

4.1 Overall reconstruction errors - choice of the reconstruction grid

How reconstruction results depend on the grid dimension is studied by varying peak extensions in each ensemble individually, for 300 random samples in each ensemble. Figure 2 shows ensemble mean values of the nearness for geometry $3 \mathrm{~T} 90^{\circ}$ of Fig. 1c and a regular grid of linear basis functions (note that for $n_{x} \times n_{y}$ pixels the dimension of the state vector is $\left(n_{x}+1\right) \times\left(n_{y}+1\right)$ for bilinear discretisation). For one peak results for both SIRT and ART are shown, where $\boldsymbol{x}^{(0)}=0$. SIRT produces smaller overall reconstruction errors, especially for narrow peaks. For all peaks except the large ones from ensemble 4 the nearness can be considerably reduced by choosing grids that lead to highly under-determined systems. This remains true even for four peaks (maxima here and in the following vary between 0.1 and 1 a.u.). For peaks 


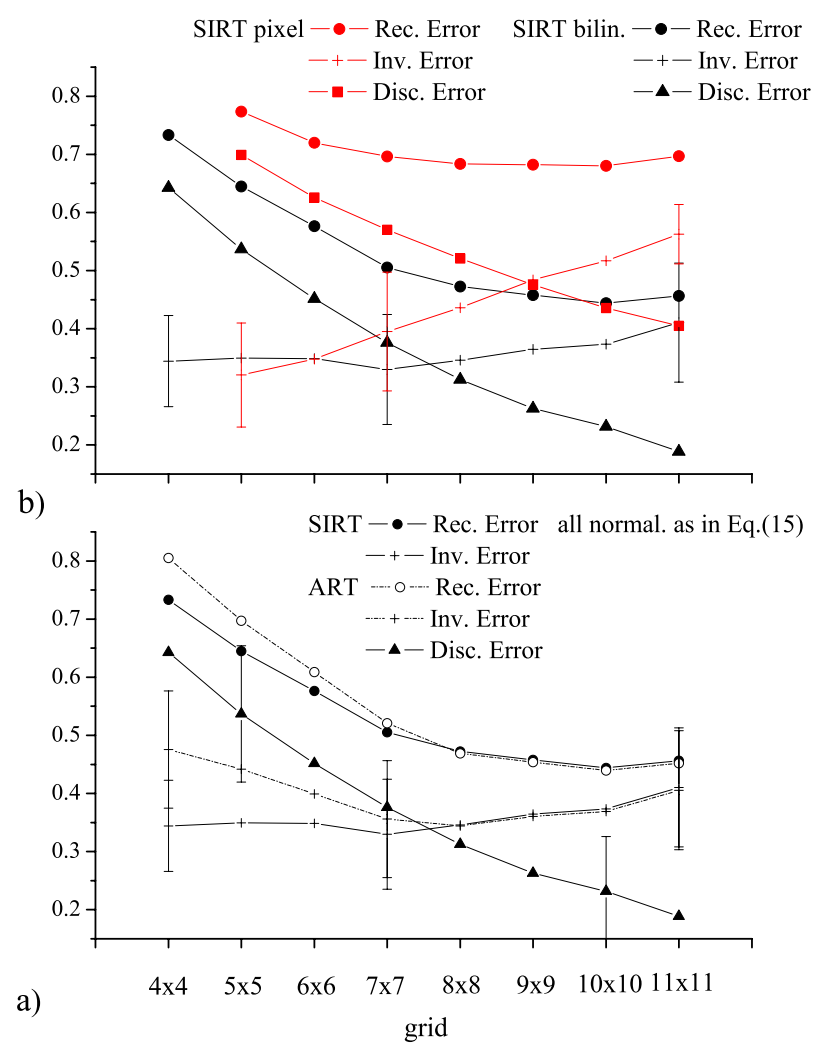

Fig. 3. Integrated reconstruction (nearness), inversion and discretisation error as defined in Eqs. (11) and (13) (without measurement noise) and normalised as in Eq. (15) for four peaks from ensembles 1 and 2. Peak heights vary between 0.1 and 1 a.u. (again $n \times n$ means $\operatorname{dim}(\boldsymbol{x})=(n+1) \times(n+1)$ in the bilinear case). (a) ART and SIRT. (b) Pixel and linear discretisation for the same dimension of the state vector.

from ensemble 3 and 4 the averaged mean concentration of all light paths is taken as a priori. Approaching the overdetermined region, differences between SIRT and ART grow, especially for the more narrow peaks. Indeed, in these cases the optimal iteration number lies before convergence and reconstruction errors increase with iteration number, which either means that the least squares minimum norm solution has to be regularised or that it is not a good solution.

A grid of $10 \times 10$ pixels means three times more unknowns than knowns in the Eq. (3). Three reasons make the highly under-determined case still better for narrow peaks. First, most of the grid nodes carry the concentration zero and this is just provided by the a priori. Second, the positivity constraint becomes active for solutions around zero. Finally, the discretisation error decreases with increasing grid dimension. This is illustrated by Fig. 3, where total reconstruction, inversion and discretisation errors according to Eqs. (11) and (13) and normalised as in Eq. (15) are shown for four peaks from ensembles 1 and 2, comparing ART and SIRT (a) and box and linear basis functions (b). As can be seen from the in-

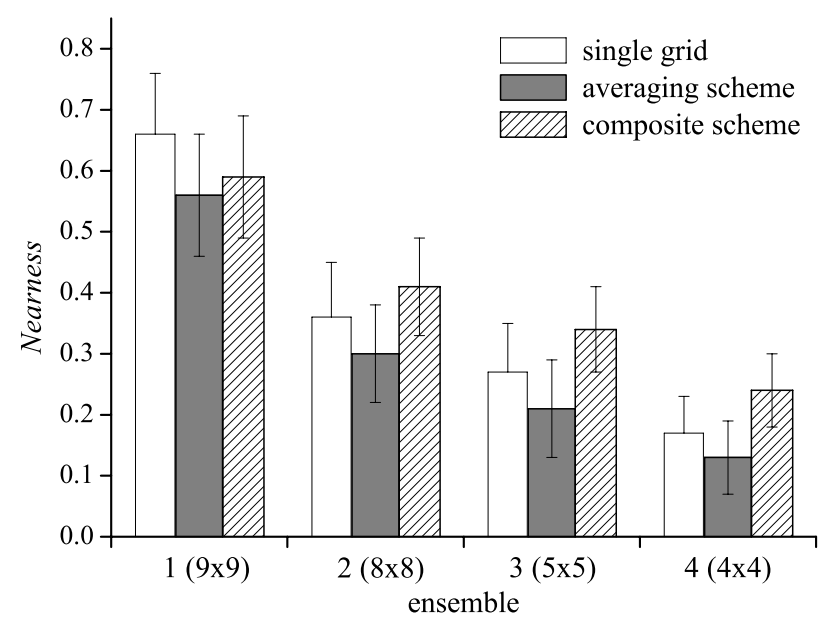

Fig. 4. Mean nearness for reconstruction of four peaks from ensembles 1 to 4, respectively, for the averaging and composite grid translation scheme. In both cases the original grid was shifted four times in each direction. The bilinear grids used for reconstruction with SIRT are given in brackets.

version error, the decreasing nearness for higher dimensional grids is finally solely due to decreasing discretisation errors. Taking the inversion error as a measure for the quality of the solution of Eqs. (4), (5), and (6) this suggests that minimising nearness is not sufficient to find the optimal grid. This agrees with the fact that artefacts in the reconstruction increase for grids too fine, even if the nearness still decreases. Figure $3 \mathrm{~b}$ compares pixel and linear discretisation for reconstruction by SIRT. Not only is the discretisation error larger than in the linear case but also the inversion error. This is not at all evident and cannot just be explained by different conditioning of the corresponding matrices, at least not in terms of mere condition numbers. We conclude that the linear discretisation is not just smoothing the picture but actually more accurate, at least for the case shown here.

For the sake of completeness, we remark that data residuals show a different behaviour in that they always decrease with increasing grid dimension, irrespective of the extension of the peak. This is just underlining the well known fact that the data residual is only of very limited use for evaluating a reconstruction result. Here, relative residuals for one peak from ensemble 1 are below $10 \%$, below $5 \%$ for ensemble 2 and far below $1 \%$ for ensembles 2 and 3 .

Figure 4 shows how mean nearness is changed by applying the two schemes of shifted grids to four peaks from ensembles 1 to 4, respectively. In both cases the original grid is shifted four times in each direction. Further shifts do not improve nearness. The averaging scheme improves the nearness for all peak extensions between $15 \%$ and $25 \%$, whereas the composite scheme improves it only for the very narrow peaks (ensemble 1) and deteriorates it for larger. In Verkruysse and Todd (2004) a scheme similar to 
the composite scheme used here was found to improve nearness for the box basis functions.

Figure 5 shows reconstruction examples for peaks from each ensemble and illustrates how results for narrow peaks depend on the grid chosen. Larger pixels lead to smaller peak concentrations; the averaging grid translation scheme does not in general seem to diminish artefacts and can indeed lead to worse reconstruction results in terms of nearness if the original grid happens to be above the average of the shifted grids. In Fig. 5a grids of higher dimension give higher peak values and somewhat smaller peak diameters, but without increasing artefacts. This could be seen as an indication for a very narrow original peak and thus justify the choice of a very high dimensional grid. Figure $5 \mathrm{~b}$, on the other hand, shows how artefacts appear in the reconstruction if the grid is too fine. Finally, Fig. 6 presents reconstructions of two and four peaks. Reconstruction by the single, unfavourable $8 \times 8$ grid in Fig. 6a gives a misleading picture that can be corrected by comparison with other grids or by using the averaging grid translation scheme. Figure $6 b$, too, illustrates how accidental features are corrected by averaging over grids.

\subsection{Reconstructed area integrals}

The same trend with grid dimension found for the integrated reconstruction error exists for area integrals of the reconstructed concentration and the differences of the maxima. Figure 7 shows the results for the geometry and test distributions of the previous section. Ensemble mean absolute deviations of the reconstructed concentration integrals over the whole area RTI, c.f. Eq. (16), (Fig. 7b) and within the plumes RPI, Eq. (17), (Fig. 7a) crucially depend on peak extensions. For optimal grids the concentration integrals over the whole area deviate between 10 and $35 \%$ for one narrow peak from the original test field, between 5 and $20 \%$ if four narrow peaks are reconstructed and less than 5 and $10 \%$, respectively, for extended distributions. For the concentration integrated over the area within the plume, one gets mean deviations up to $20 \%$ for one narrow peak, up to $30 \%$ for four peaks and less than 5 and $10 \%$ for larger plumes. The steep decrease with increasing grid dimension for narrow peaks is not simply due to the smaller pixel size, as this is taken into account in the plume extension as described in Sect. 2.5. In general, both integrals can be over- or underestimated, depending on the location and relative values of the peak maxima. While ART and SIRT yield the same plume integrals for one peak, ART shows larger deviations for the area integrals, agreeing with the visual impression that it produces more artefacts in the reconstruction. In this context, we would like to point out that the total area integral increases if a concentration peak is spread over the area and the column densities are kept constant.

The averaging grid translation scheme does not significantly change integrated concentrations, whereas the com- posite scheme yields much larger deviations of the full area integrated concentration, especially for narrow peaks (figures not shown). Plume integrals of concentration remain largely unaltered (also not shown). The maximum peak difference (Fig. 7c) is strongly reduced for narrow peaks by choosing a finer discretisation as the concentration is spread over smaller pixels and the peak values increase. Applying the averaging grid translation scheme leads to an increased deviation by 30,30 and $20 \%$ for ensembles 1, 2 and 3 and practically no change for ensemble 4 . In contrast to the case of box basis functions (Verkruysse and Todd, 2004), the composite scheme reduces the peak difference only for ensemble 1 (by $50 \%$ ). So by the grid translation schemes one can obtain better results for narrow peaks in terms of nearness as overall deviation of the shape, but not in terms of peak concentrations and concentration integrals (c.f. Fig. 5). As reconstruction of the precise shape of the distribution is hardly possible for very narrow peaks and the geometry considered here, the question, whether grid translation should be used depends on the features most important to be reconstructed correctly.

\subsection{Additional background concentration}

As pointed out above, one reason that makes a fine discretisation and thus a highly under-determined system feasible is that the a priori $\boldsymbol{x}^{(0)}=0$, supported by the positivity constraint, is a good choice for narrow peaks. In the presence of background concentrations this no longer holds and the a priori has to be modified. If the background distribution is more or less smooth and not of particular interest or even known, we suggest subtracting a contribution given by the background concentration $d_{i}^{B G}=\int_{i} d s c^{B G}(\mathbf{r})$ and solving the resulting system $\boldsymbol{d}-\boldsymbol{d}^{B G}=\mathbf{A} \boldsymbol{y}$ again with a priori as in Sect. 4.1. Here, we substract a constant background $c^{B G}$ given by the smallest column density $d_{\min }=: c_{B G} l_{\min }$ ( $l_{\min }$ being the length of this light path). This is not equivalent to taking $c^{B G}$ as a priori - not even in the linear case - and yields better results in terms of nearness than using the original system with a non-zero a priori, like e.g. averaged mean concentration of all light paths, as shown in Fig. 8. In this figure both methods are compared for a randomly created background added to four peaks from ensembles 1, 2, 3 and 4, respectively. Concentrations of the background vary between 0.05 and 0.25 a.u., peak maxima between 0.1 and 1 a.u. so that the contributions from the background to the total column densities are similar in size to those from the peaks. The reconstruction error decreases mainly for ensembles 2 and 3, only slightly for ensemble 1 and increases slightly for large distributions from ensemble 4.

\subsection{Different geometries}

The mathematically ideal measurement would be represented by a diagonal matrix $\mathbf{A}$ with entries of similar size. For a regular box discretisation this would mean one light path 
a) Test distribution

Single grid: $9 \mathrm{x} 9$

$($ Nearn $=0.6, \mathbf{R P I}=1.1, \mathbf{R T I}=1.3)$

$10 \times 10$

$(0.5,1.1,1.3)$
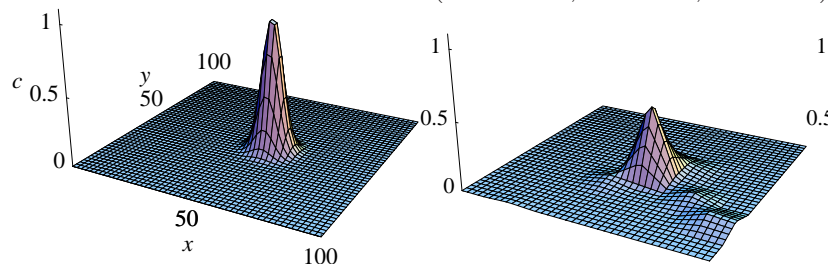

Grid translation: 9x9 $(0.5,1.1,1.3)$

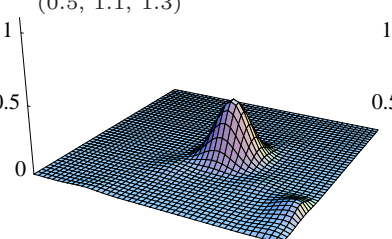

b) Test distribution

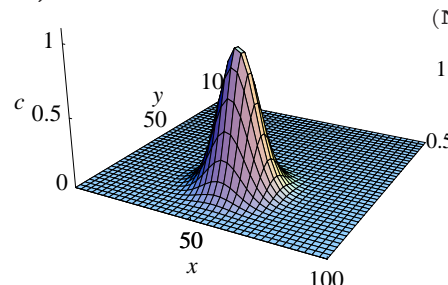

Single grid: $8 \times 8$

$($ Nearn $=0.31, \mathbf{R P I}=1.0, \mathbf{R T I}=1.1)$

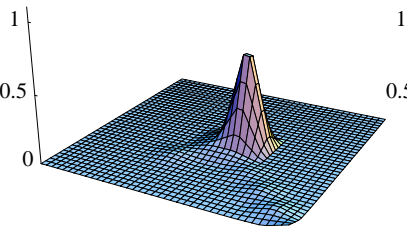

$10 \times 10$

$(0.42,1.1,1.3)$
$12 \times 12$

$(0.29,1.0,1.1)$

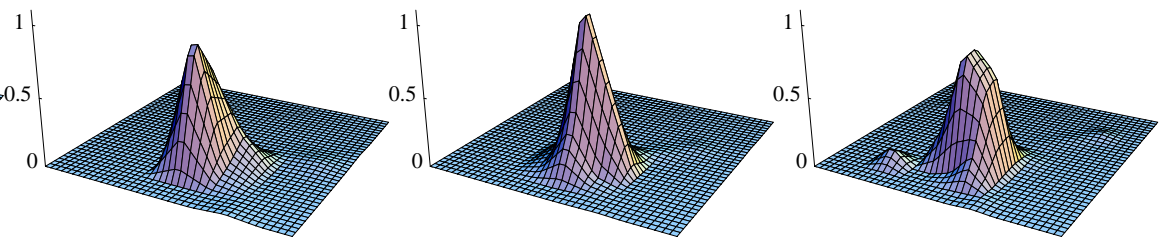

Grid translation: $8 \times 8$

$10 \times 10$

$12 \times 12$

$(0.16,1.0,1.1)$

$(0.06,1.0,1.0)$

$(0.13,1.0,1.0)$

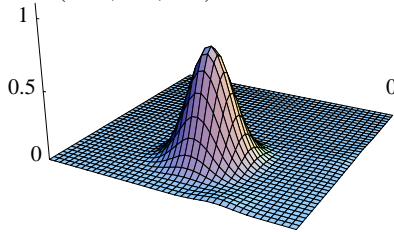

$(0.06,1.0,1.0)$
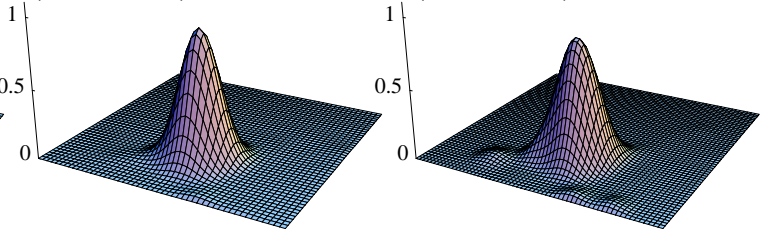

c) Test distribution

Single grid: $5 \times 5$

d) Test distribution

Single grid: $4 \mathrm{x} 4$

$($ Nearn $=0.18, \mathbf{R P I}=0.95, \mathbf{R T I}=0.95)$
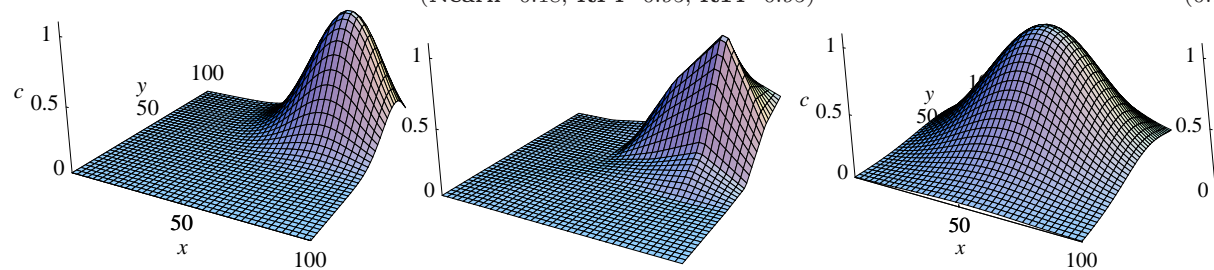

$(0.14,1.0,1.0)$

Grid translation: $5 \times 5$

$$
(0.13,0.8,1)
$$

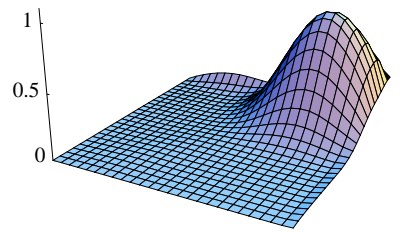

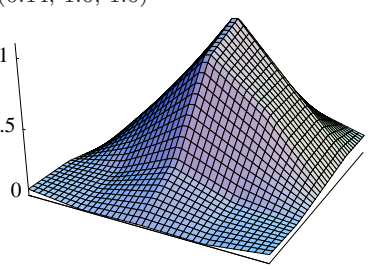

Grid translation: $4 \times 4$

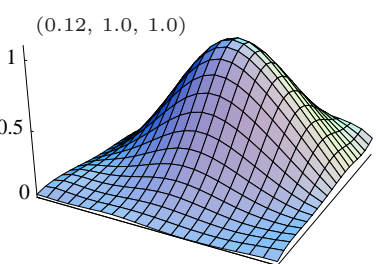

Fig. 5. Reconstruction of one peak by SIRT using a single, regular, bilinear grid and the averaging grid translation scheme, where the original grid is shifted four times in each direction. Peak from (a) ensemble 1 ( $\sigma=4$ a.u.), (b) ensemble 2 ( $\sigma=7$ a.u.), (c) ensemble 3 ( $\sigma=15$ a.u.), (d) ensemble 4 ( $\sigma=25$ a.u.). 
a) Test distribution

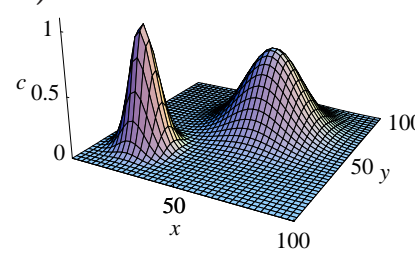

Grid translation: $10 \times 10$

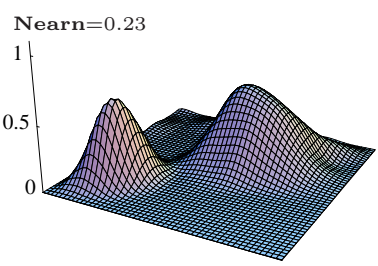

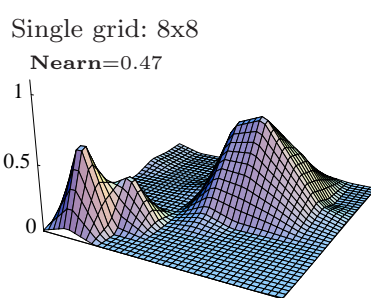

b) Test distribution

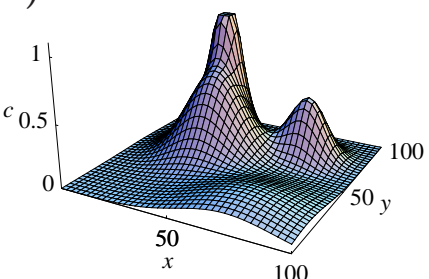

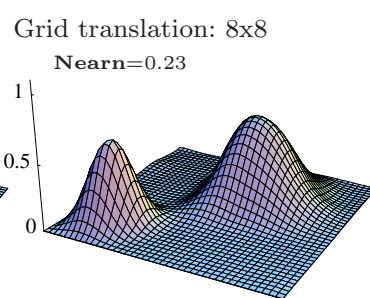

Single grid: $8 \times 8$

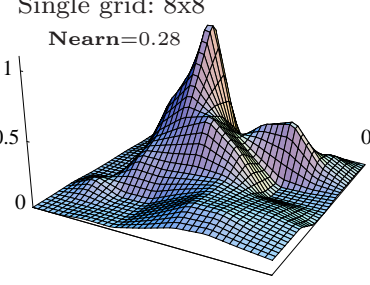

Single grid: $10 \times 10$ Nearn $=0.26$

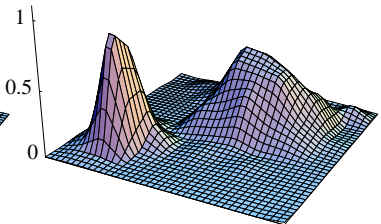

Grid translation: $8 \times 8$

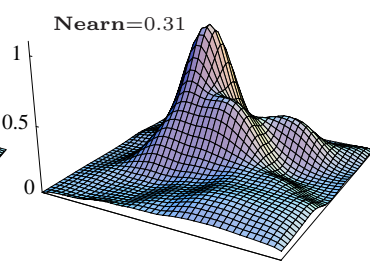

Fig. 6. Same as Fig. 5. (a) Two peaks with $\sigma_{1,2}=13,6$ a.u. and $c_{\max 1,2}=0.7,1$ a.u. (b) Four peaks with $\sigma_{1,2,3,4}=13,6,8,20$ a.u. and

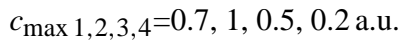

in each pixel, i.e. as many telescopes as pixels, resulting in maximum independency of the light paths. Light paths get more independent if emitted by more telescopes or in wider angles. The expectation that increasing the number of telescopes and using e.g. $180^{\circ}$-fans instead of $90^{\circ}$-fans improves reconstruction results was confirmed in Todd and Bhattacharyya (1997) for a far larger number of light paths and slightly different geometries including projections. For the geometries in Fig. 1, one expects improving reconstructions going from two to four telescopes, but worse results for the $180^{\circ}$-geometries compared to those with $90^{\circ}$-fans due to their coarser and less regular coverage with light paths. This is shown in Fig. 9a, where mean nearness is calculated for four peaks from ensembles 1 to 3 for the different geometries using SIRT on an $8 \times 8$ grid and bilinear basis functions without grid translation scheme. Also shown is the Pearson $r^{2}$-correlation between original and reconstructed picture for $50 \times 50$ sample points with mean values between 0.7 and 0.88 . The $90^{\circ}$-geometry with two telescopes gives by far the largest reconstruction errors. Though it does get better for narrow distributions, the overall order of the geometries does not depend very much on the ensembles chosen. Reconstruction quality varies strongly with the measurement geometries and number of peaks as indicated for the $90^{\circ}$-geometry with three telescopes. Reconstructed area integrals of the concentration field within the peaks ( $R P I$ ) (Fig. 9b) deviate around $5 \%$ in the ensemble mean from real ones for the geometries with more than two telescopes and around $10 \%$ for two telescopes with distinctly larger ensemble variances. The same trend with geometry as for nearness and RPI holds for the complete area integrated concentrations RTI (Fig. 9c) and for the relative peak difference of the maximum peaks (Fig. 9d).

To get an idea of how the reconstruction quality varies within the test area, quality criteria are evaluated for one peak on a fine grid of peak positions and mapped to the area by displaying the value of the criterion at the position of the peak in the reconstruction area (Fig. 10). In the first, second and third column nearness, $R P I$ and $R T I$ are given for one peak from ensemble 2 ( $\sigma=7$ a.u.), reconstructed on an $8 \times 8$ linear grid for $30 \times 30$ peak positions without using any grid shifting scheme. Apart from the pattern of smaller nearness values if peak positions coincide with grid nodes, there are spots of larger reconstruction errors where coverage with light paths is coarse. The maps of the concentration integrals within the whole area and within the peak, respectively, show this pattern, too. Both get underestimated in gaps between light paths and overestimated around the telescopes where only similar light paths from the same fan contribute. Taking the plots for RPI and RTI together gives information about where the reconstructed trace gas is located: if the deviation for the concentration integrated over the whole area is distinctly larger than within the peak, then the distribution is spread over a larger region (as is the case near the telescopes) or artefacts appear somewhere else (which here, for one peak, is rarely the case).

Finally, the fourth column of Fig. 10 illustrates how well concentration values are fixed by the data according to Sect. 3, again for an $8 \times 8$ grid. The interpolating representation is slightly misleading in that values of $\sum_{j>\operatorname{rank}(\mathbf{A})} V_{i j}{ }^{2}$ exist only on the nodes $i$. How much the null space actually contributes depends on the singular vector representation of the state vector given. Even for high values of $\sum_{j>\operatorname{rank}(\mathbf{A})} V_{i j}{ }^{2}$, the null space does not contribute if corresponding components $\tilde{x}_{i}$ vanish, but for a statistical average of arbitrary concentration distributions, these maps should point out weak points of an irregular geometry and indeed do so, as can be seen from comparison with the first three columns, especially for geometries $2 \mathrm{~T} 90^{\circ}$ and $2 \mathrm{~T} 180^{\circ}$.

The evaluation in Fig. 10 was made for the special shape of Gaussian distributions and without taking measurement 


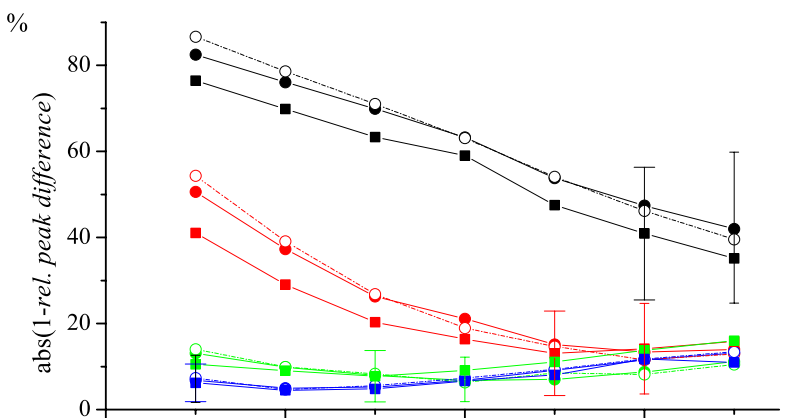

c)

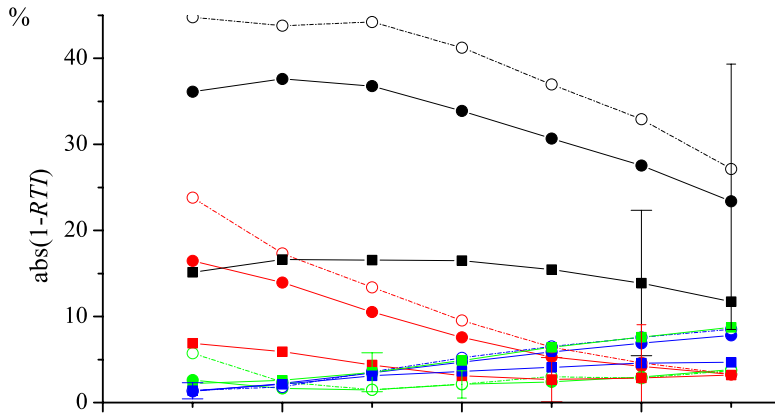

b)

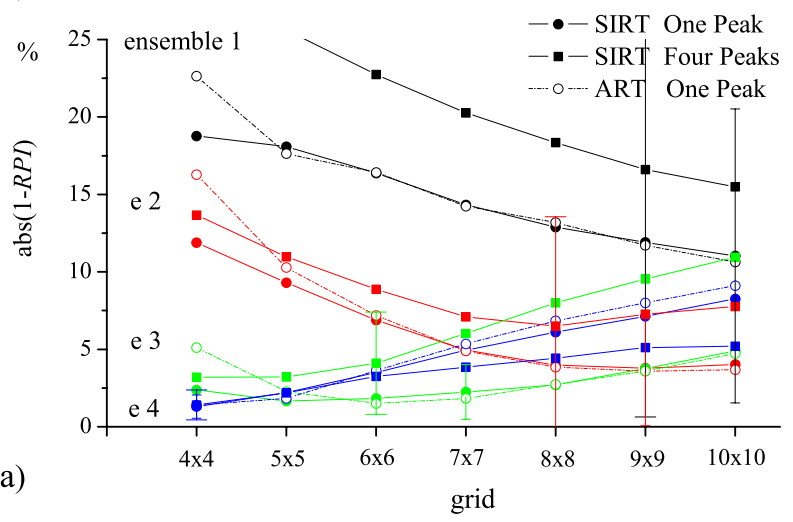

Fig. 7. Ensemble mean deviations of (a) the concentration integrated within the peak $(R P I)$, (b) the concentration integrated over the whole area $(R T I)$ and (c) the relative peak difference of the maximum peaks between the reconstructed and original distribution. Settings are as in Fig. 2, variances shown where nearness has its minimum and for SIRT only.

errors into account. To see how the geometries perform for different levels of noise, Figs. 11 and 12 show the number of degrees of freedom of the signal and information content, respectively, for different a priori assumptions on the trace gas distribution to be measured. In Fig. 11a and 12a uniform a priori covariance $\mathbf{S}_{\mathbf{a}}=\operatorname{diag}\left(\sigma_{a}, \ldots, \sigma_{a}\right)$ and uncorrelated measurement errors of the same size $\sigma_{\varepsilon}$ were assumed. For the distributions and areas considered here, ratios $\sigma_{a} / \sigma_{\varepsilon} \approx 1$ correspond to measurements with relative errors of a few percent. For negligible measurement errors, all geometries use their maximum degree of freedom of all 36 light paths

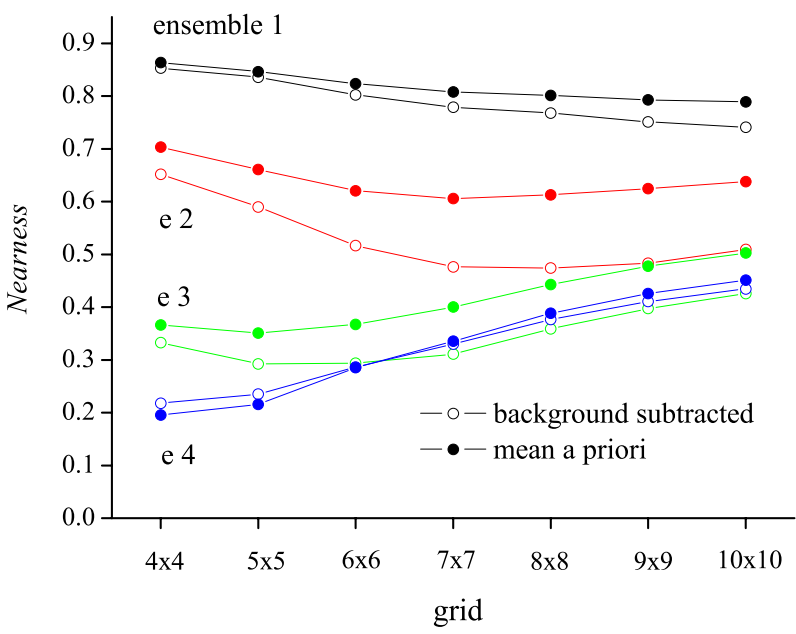

Fig. 8. Ensemble mean nearness for four peaks with background reconstructed after subtracting the background and by assuming the averaged mean concentration of all light paths as a priori. Peak maxima vary between 0.1 and 1 a.u. and the randomly created background between 0.05 and 0.25 a.u. No grid shift scheme is applied.

$\left(\sigma_{\varepsilon} / \sigma_{a} \rightarrow 0\right.$ corresponds to reconstruction by the generalised inverse). The geometries with only two telescopes are very sensitive to noise, whereas realistic measurements with three and four telescopes still almost reach their maximum degree of freedom. The information content shows a behaviour very similar to the nearness in Fig. 9a except for the high ranking of the $180^{\circ}$-geometry with four telescopes relative to those with two telescopes. This changes if the a priori covariance is restricted within the area. For the information content of the measurement in Fig. $12 \mathrm{~b} \mathbf{S}_{\mathbf{a}}$ has nonzero elements $\sigma_{a}$ on a square area of nine grid nodes only, located in the centre, the upper left and lower right corner, respectively. The geometries with two telescopes now win relatively. The centre is better resolved for all geometries except for geometry $2 \mathrm{~T} 180^{\circ}$. Here the full degrees of freedoms are not reached, as shown in Fig. 11b. For all other geometries measurements in the centre are less sensitive to noise.

\section{Conclusions}

In this study, a discrete approach to retrieve trace gas distributions from Long path DOAS-measurements was investigated in detail with respect to the parametrisation of the solution and the extension of the concentration distribution. We found that, at least for the reconstruction of concentration peaks by the least squares minimum norm solution with additional positivity constraint, parametrisation by piecewise linear basis functions instead of piecewise basis functions not only reduces the discretisation error but also improves the inversion itself. Reconstruction errors and the inaccuracy of reconstructed total amounts of trace gas concentrations can 
c)

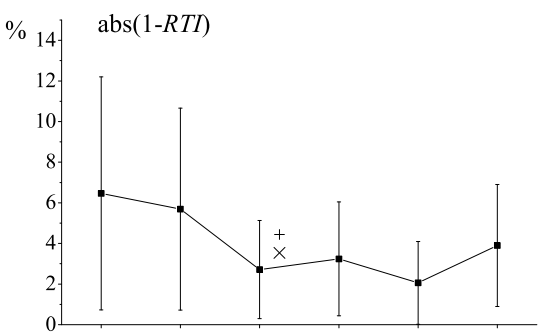

a)
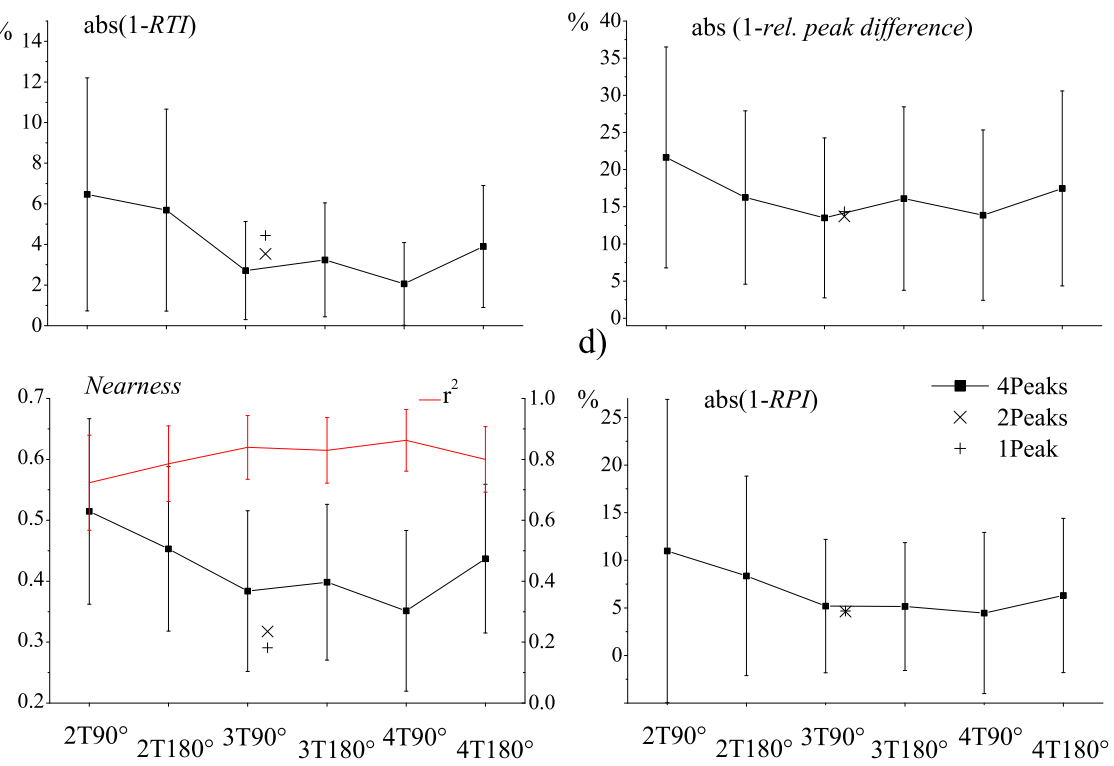

b)

Fig. 9. Ensemble mean values for four peaks from ensembles 1 to 3 for the geometries of Fig. 1. (a) Nearness and Pearson $r^{2}$-correlation between original and reconstructed picture for $50 \times 50$ sample points. (b) Deviation of the concentration integrated within the plume $(R P I)$. (c) Deviation of the concentration integrated over the whole area $(R T I)$. (d) Relative peak difference of the maximum peaks. For geometry $3 \mathrm{~T} 90^{\circ}$ values for one and two peaks are also shown. Reconstruction used SIRT on an $8 \times 8$ grid without grid shifting scheme.

be significantly reduced by choosing an optimal dimension of the discretisation grid. A grid translation scheme adopted from Verkruysse and Todd (2004) that averages reconstruction results of several discretisation grids shifted against each other reduces total reconstruction errors and accidental features of a single reconstruction grid and can therefore help with the a posteriori choice of the discretisation grid. It does not in general lead to better estimation of total amounts of concentration and underestimates peak maxima. The results hold for a limited number of isolated peaks but can be extended to the case of additional smooth background if subtracted before reconstruction.

Reconstruction quality for a given number of light paths strongly depends on the beam configuration. Increasing the number of telescopes for the same number of light paths leads to better reconstruction results, provided that the coverage with light paths can be kept regular and does not decrease. Measurement errors have less impact on better geometries but the sensitivity varies within the area and seems to be weaker for better resolved regions.

The minimum norm solution combined with appropriate a priori seems to be suitable for the test distributions considered here but has the major drawback of being nonlinear due to the additional positivity constraint. Linear reconstruction methods like the LTD method suggested in Price et al. (2001) not only are much faster, but allow analytic propagation of measurement errors and using tools from linear inversion theory like the resolution matrix, independently of specific distributions. In the over- or well-determined case, the least square minimum norm solution is completely fixed by the data and if regularisation is not necessary, introduction of any a priori should be well justified, especially if unphysical.

Furthermore, we would like to point out that all variances shown always contain the full variability within the test area and should not be taken as reconstruction errors of a particular distribution. To estimate the actual reconstruction error field from a specific set of measurement data as done by Laepple et al. (2004), only realistic distributions compatible with the uncertainty of the reconstruction should be contained in the ensemble for the error estimation. On the other hand, these test distributions should reproduce the temporal and spatial variability of the atmosphere - something not true for models, like simple Gaussian dispersion models, that do not treat turbulent processes adequately. A complete error analysis should also include effects due to non-static conditions during a measurement cycle.

Finally, we stress the fact that we did not directly compare the minimum norm solution to solutions from other reconstruction principles, e.g. which use a weighted quadratic constraint on the state vector norm or its derivatives. But on the basis of our findings, we think that such a comparison should be made with special respect to extension and gradients of the concentration fields and should use less ideal test distributions than Gaussian peaks, especially for the evaluation of grid translation schemes. 

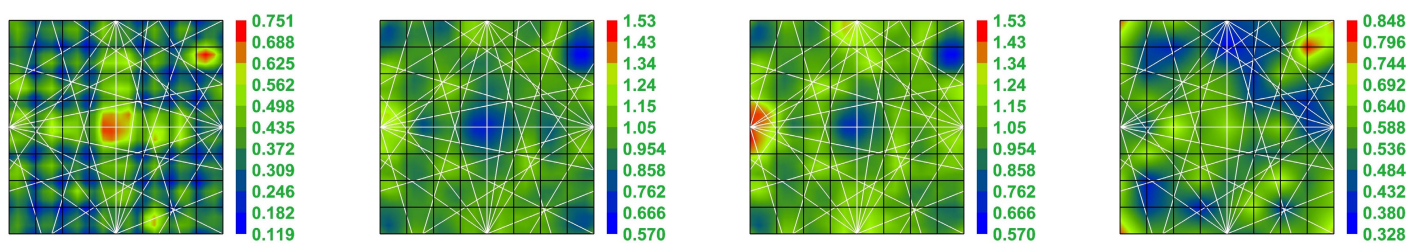

$4 \mathrm{~T} 180^{\circ}$

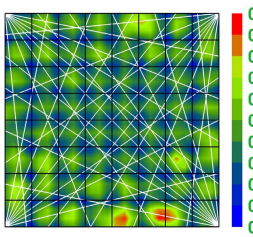

0.618
0.568
0.518
0.468
0.418
0.368
0.318
0.268
0.218
0.168
0.118
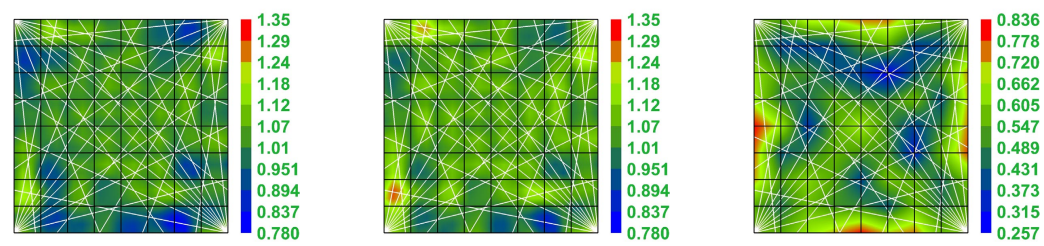

$4 \mathrm{~T} 90^{\circ}$
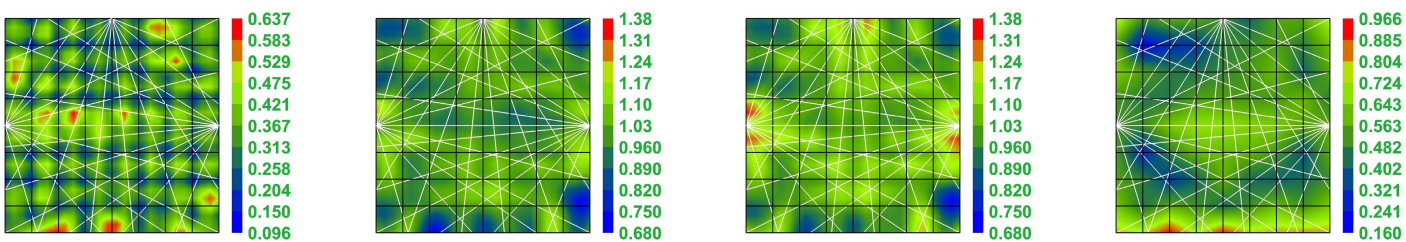

$3 \mathrm{~T} 180^{\circ}$
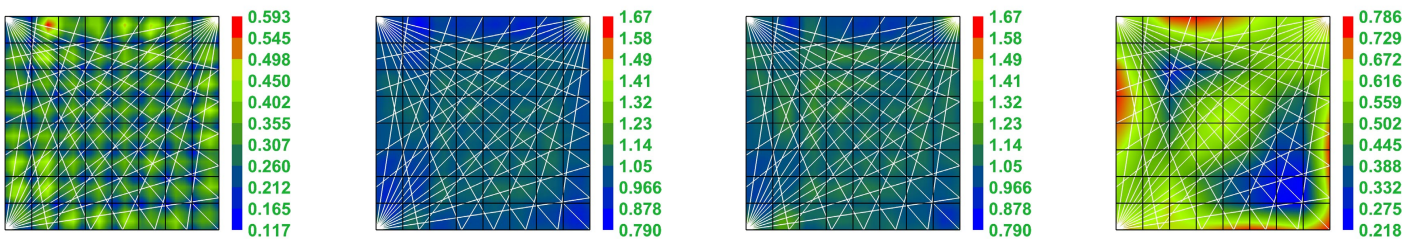

$3 \mathrm{~T} 90^{\circ}$
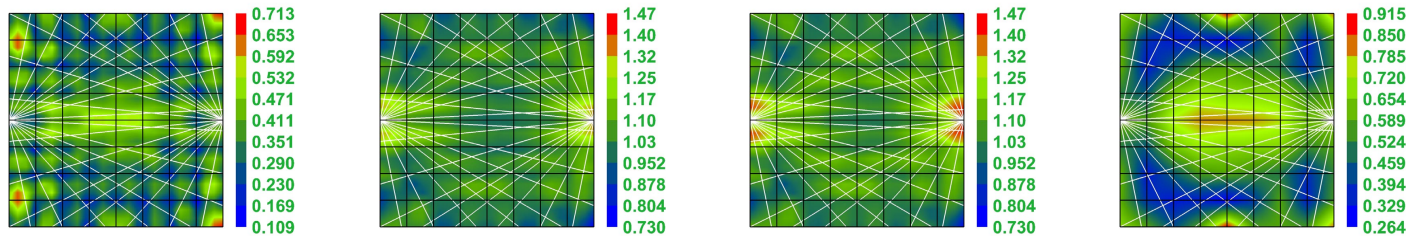

$2 \mathrm{~T} 180^{\circ}$
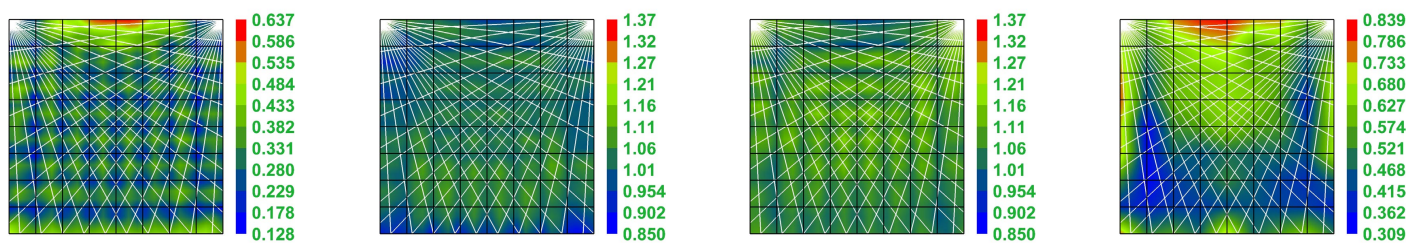

\section{T90 $\quad$ Nearness}

$R P I$

$R T I$

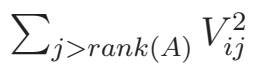

Fig. 10. First, second and third column: Quality criteria as function of peak position for reconstruction of a single peak ( $\sigma=7$ a.u.) on an $8 \times 8$ linear grid without grid shifting scheme for different geometries. First column: nearness. Second column: concentration integrated within the peak (RPI). Third column: concentration integrated within the whole area $(R T I)$. Fourth column: components $i$ of the sum $V_{i j}^{2}$ over the null space of A mapped to the nodes of the $8 \times 8$ linear grid. Values in between the grid nodes are merely interpolated. 
A. Hartl et al.: 2-D reconstruction of atmospheric concentration peaks

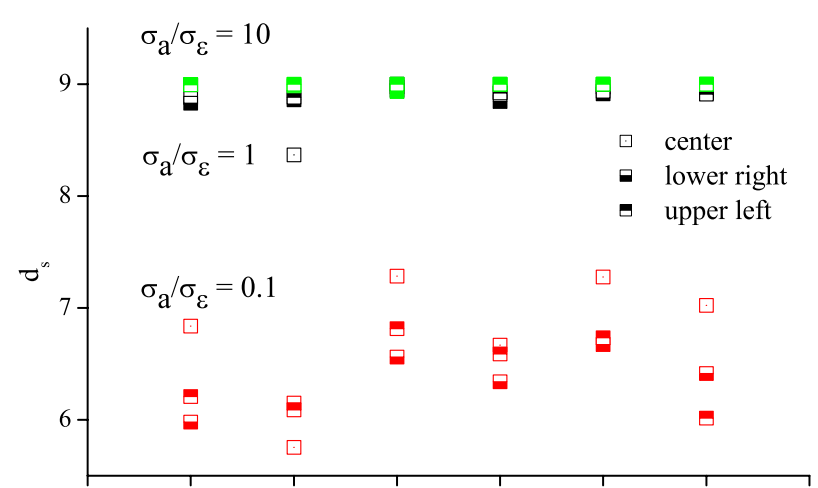

b)

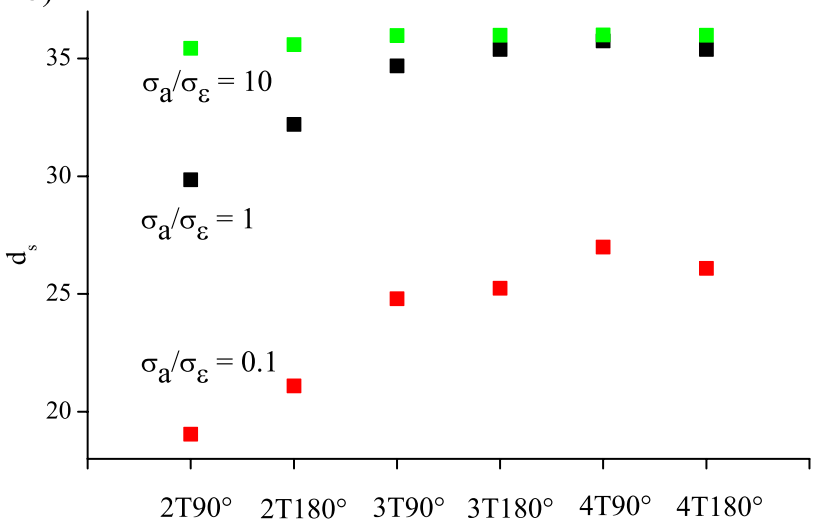

a)

Fig. 11. Degrees of freedom of the different geometries for an $8 \times 8$ linear grid and different levels of noise. (a) Uniform, diagonal a priori covariance with elements $\sigma_{a}$. (b) Diagonal a priori covariance with $\sigma_{a}$ non-vanishing on nine grid points only (corresponding to a square area of $25 \times 25$ a.u.), located in the centre, lower right and upper left corner, respectively. In all cases the measurement errors were assumed to be uncorrelated and of the same size $\sigma_{\varepsilon}$.

Acknowledgements. The German Ministry of Research and Education (BMBF) is gratefully acknowledged for the funding of this work through project 07 ATC-03 (Young researchers fellowship program for research groups, AFO 2000-C).

Edited by: G. Vaughan

\section{References}

Drescher, A. C., Gadgil, A. J., Price, P. N., and Nazaroff, W. W.: Novel approach for tomographic reconstruction of gas concentration distributions and simulated annealing, Atmos. Environ., 30, 929-940, 1996.

Drescher, A. C., Park, D. Y., Yost, M. G., Gadgil, A. J., Levine, S. P., and Nazaroff, W. W.: Stationary and time-dependent indoor tracer-gas concentration profiles measured by OP-FTIR remote sensing and SBFM-computed tomography, Atmos. Environ., 31, 727-740, 1997

Fischer, M. L., Price, P. N., Thatcher, T. L., Schwalbe, C. A., Craig, M. J., Wood, E. E., Sextro, R. G., and Gadgil, A. J.: Rapid mea-
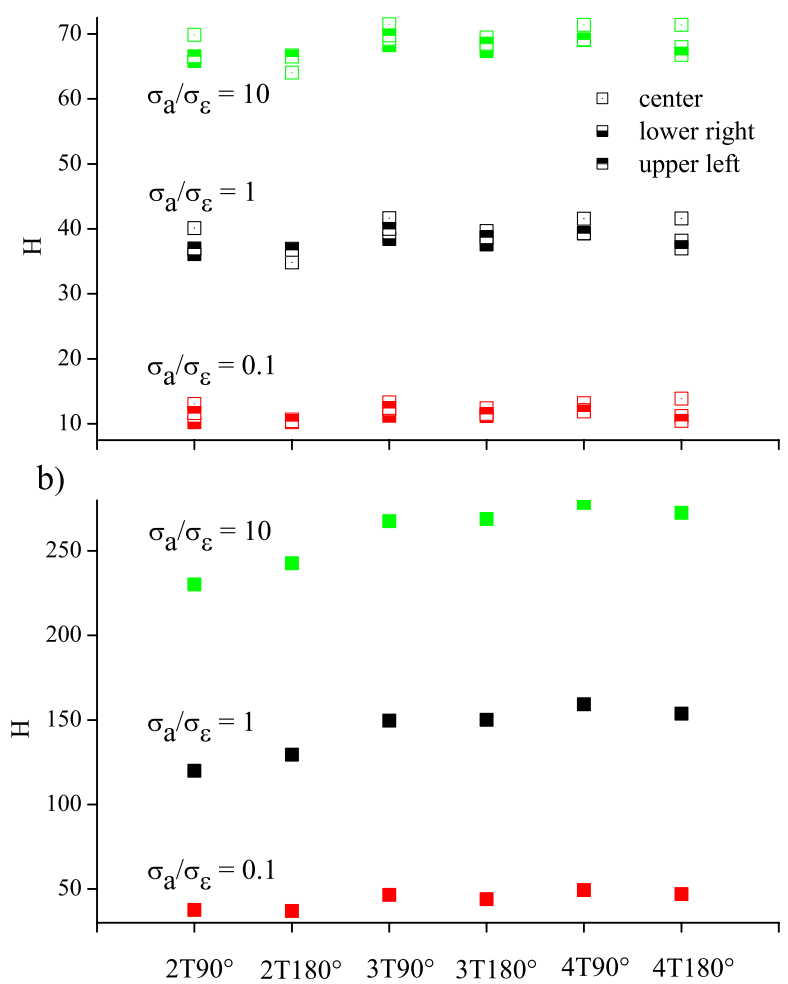

a)

Fig. 12. As Fig. 11 for the information content.

surements and mapping of tracer gas concentrations in a large indoor space, Atmos. Environ., 35, 2837-2844, 2001.

Ingesson, L. C., Alpher, B., Chen, H., Edwards, A. W., Fehmers, G. C., Fuchs, J. C., Giannella, R., Grill, R. D., Lauro-Taroni, L., and Romanelli, M.: Soft $\mathrm{X}$ ray tomography during ELMs and impurity injection in jet, Nucl. Fusion, 38, 1675-1694, 1998.

Laepple, T., Knab, V., Mettendorf, K.-U., and Pundt, I.: Longpath DOAS tomography on a motorway exhaust gas plume: numerical studies and application to data from the BABII campaign, Atmos. Chem. Phys., 4, 1323-1342, 2004.

Mettendorf,K. U., Hartl, A., and Pundt, I.: An indoor test campaign of the tomography long path differential optical absorption spectroscopy technique, J. Environ. Monit., 8, 1-9, 2006.

Natterer, F.: The mathematics of computerized tomography, Stuttgart, Teubner, New York, Wiley, 1986.

Platt, U.: Air monitoring by Differential Optical Absorption Spectroscopy (DOAS), in: Air Monitoring by Spectroscopic Techniques, edited by: Sigrist, M. W., Chemical Analysis Series, vol. 127, John Wiley \& Sons, Inc., New York, 1994.

Pundt, I., Mettendorf, K.-U., Laepple, T., Knab, V., Xie, P., Lösch, J., v. Friedeburg, C., Platt, U., and Wagner, T.: Measurements of trace gas distributions by Long-path DOAS-tomography during the motorway campaign BAB II: experimental setup and results for $\mathrm{NO}_{2}$, Atmos. Environ., 39, 967-975, 2005.

Pundt, I. and Mettendorf, K.-U.: Multibeam long-path differential optical absorption spectroscopy instrument: a device for simultaneous measurements along multiple light paths, Appl. Opt., 44, 4985-4994, 2005. 
Price, P. N., Fischer, M. L., Gadgil, A. J., and Sextro, R. G.: An algorithm for real-time tomography of gas concentrations using prior information about spatial derivatives, Atmos. Environ., 35, 2827-2835, 2001.

Rodgers, C. D.: Inverse methods for atmospheric sounding: Theory and Practice, World Scientific Publishing Co., Singapore, 2000.

Samanta, A. and Todd, L. A.: Mapping chemicals in air using an environmental CAT scanning system: evaluation of algorithms, Atmos. Environ., 34, 699-709, 2000.

Todd, L. and Ramachandran, G.: Evaluation of algorithms for tomographic reconstruction of chemical concentrations in indoor air, Am. Ind. Hyg. Assoc. J., 55, 403-416, 1994a.

Todd, L. and Ramachandran, G.: Evaluation of optical sourcedetector configurations for tomographic reconstruction of chemical concentrations in indoor air, Am. Ind. Hyg. Assoc. J., 55, 1133-1143, 1994b.

Todd, L. and Bhattacharyya, R.: Tomographic reconstruction of air pollutants: evaluation of measurement geometries, Appl. Opt., 36, 7678-7688, 1997.
Trampert, J. and Leveque, J.-J.: Simultaneous Iterative Reconstruction Technique: Physical interpretation based on the generalised least squares solution, J. Geophys. Res., 95, 12553 $12559,1990$.

Twomey, S.: Introduction to the mathematics of inversion in remote sensing and indirect measurements, Dover Publications Inc, Mineola, New York, 1996.

Verkruysse, W. and Todd, L. A.: Improved Method "grid translation" for mapping environmental pollutants using a twodimensional CAT scanning system, Atmos. Environ., 38, 18011809, 2004.

Wolfe, D. C. and Byer, R. L.: Model studies of laser absorption computed tomography for remote air pollution measurement, Appl. Opt., 21, 1165-1177, 1982.

Yost, M. G., Gadgil, A. J., Drescher, A. C., Zhou, Y., Simonds, M. A., Levine, S. P., Nazaroff, W., and Saisan, P.: Imaging indoor tracer-gas concentrations with computed tomography: experimental results with a remote sensing FTIR system, Am. Ind Hyg. Ass. J., 55, 395-402, 1994. 